OPEN ACCESS

Edited by:

Ignacio Sanz,

University of Rochester, USA

Reviewed by:

Paulo Vieira,

Institut Pasteur de Paris, France

Paolo Casali,

University of Texas School

of Medicine, USA

Joshy Jacob,

Emory University, USA

*Correspondence:

Gunilla B. Karlsson Hedestam gunilla.karlsson.hedestam@ki.se

Specialty section:

This article was submitted to B Cell Biology,

a section of the journal

Frontiers in Immunology

Received: 20 April 2016

Accepted: 07 June 2016

Published: 27 June 2016

Citation:

Martinez-Murillo P, Pramanik L,

Sundling C, Hultenby $K$,

Wretenberg $P$, Spångberg $M$ and Karlsson Hedestam GB (2016)

CD138 and CD31 Double-Positive

Cells Comprise the Functional

Antibody-Secreting Plasma Cell

Compartment in Primate Bone

Marrow.

Front. Immunol. 7:242

doi: 10.3389/fimmu.2016.00242

\section{CD138 and CD31 Double-Positive Cells Comprise the Functional Antibody-Secreting Plasma Cell Compartment in Primate Bone Marrow}

\author{
Paola Martinez-Murillo', Lotta Pramanik', Christopher Sundling ${ }^{1,2}$, Kjell Hultenby ${ }^{3}$, \\ Per Wretenberg ${ }^{4}$, Mats Spångberg ${ }^{5}$ and Gunilla B. Karlsson Hedestam ${ }^{1 *}$
}

'Department of Microbiology, Tumor and Cell Biology, Karolinska Institutet, Stockholm, Sweden, ${ }^{2}$ Immunology Division, Garvan Institute of Medical Research, Darlinghurst, NSW, Australia, ${ }^{3}$ Division of Clinical Research Centre, Department of Laboratory Medicine, Karolinska Institutet, Huddinge, Sweden, ${ }^{4}$ Department of Orthopedic Surgery, Karolinska University Hospital, Stockholm, Sweden, ${ }^{5}$ Comparative Medicine, Astrid Fagraeus Laboratory, Karolinska Institutet, Stockholm, Sweden

Plasma cells (PCs) are defined as terminally differentiated B cells that secrete large amounts of immunoglobulin (lg). PCs that reside in the bone marrow (BM) are responsible for maintaining long-term antibody (Ab) responses after infection and vaccination, while PCs present in the blood are generally short-lived. In rhesus macaques, a species frequently used for the evaluation of human vaccines, B cells resemble those found in humans. However, a detailed characterization of BM-resident rhesus PC phenotype and function is lacking. Here, we examined Ig secretion of distinct rhesus CD138+ populations by B cell ELISpot analysis to couple phenotype with function. We demonstrate that the CD20low/-CD138+CD31+ BM population was highly enriched for antibodysecreting cells with IgG being the predominant isotype (60\%), followed by IgA (33\%) and IgM (7\%). Transmission electron microscopy analysis confirmed PC enrichment in the CD20low/-CD138+CD31+ population with cells containing nuclei with "spokes of a wheel" chromatin structure and prominent rough endoplasmic reticulum. This panel also stained human BM PCs and allowed a clear distinction between BM PCs and short-lived peripheral PCs, providing an improved strategy to isolate PCs from rhesus BM for further analysis.

Keywords: plasma cells, rhesus macaque, antibodies, elispot, bone marrow, cryopreservation

\section{INTRODUCTION}

Durable immunity relies on constitutive antibody $(\mathrm{Ab})$ production by terminally differentiated, long-lived bone marrow (BM)-resident plasma cells (PCs). These cells are products of the germinal center (GC) reaction where $B$ cells proliferate upon recognition of cognate antigen in a $\mathrm{T}$ helper cell-dependent manner. Following somatic hypermutation (SHM) of Ab variable domains, selected B cells differentiate either into memory B cells or PCs prior to exiting the GC [reviewed in Ref. (1)]. Blimp-1, along with XBP-1 and IRF-4, is a key transcription factor for the transition to fully differentiated PCs (2). 
In acute inflammatory situations, such as in response to infection or immunization, new peripheral PCs are generated. These cells are short-lived and migrate toward the inflammatory sites to secrete antigen-specific antibodies $(3,4)$. The BM is the main compartment where long-lived PCs persist in healthy individuals, and homing to this compartment is essential for long-term serological memory. This process is tightly regulated by the expression of adhesion molecules, chemokines and their receptors, where CXCL12 and its receptor, CXCR4, play major roles $(5,6)$. The BM provides a milieu that supports the persistence of long-lived PCs (7). Its cellular components consist mainly of CXCL12+VCAM+ stromal cells and hematopoietic cells $(5,8)$, including eosinophils and megakaryocytes, capable of secreting IL- 6 and APRIL that regulate the frequency and survival of PCs via their receptors IL-6R and BCMA/TACI (9-11), respectively (9-12). BAFF and APRIL signaling upregulates the expression of the anti-apoptotic molecule Mcl-1, which is essential for the long-term survival of PCs (13). In addition, the B cell intrinsic program that is imprinted during the GC reaction by intrinsic factors is indispensable for determining the fate and maintaining durable $\mathrm{Ab}$ responses $(12,14)$.

In humans, BM-resident PCs are typically defined by the expression of CD38 and CD138 as well as heterogeneous CD19 expression (15), combined with their ability to secrete $\mathrm{Ab}$ or stain positively for cytoplasmic Ig. Recent studies reported that of these PCs, the CD19- cells display a more differentiated phenotype (16) that was associated with a longer life span (17) compared to CD19+ PCs. Less information is available about BM-resident PCs in non-human primates. Rhesus macaques are frequently used to model human immunology. With the publishing of the rhesus macaque genome (18) and the development of protocols to phenotype rhesus B cell populations and PCR amplify rhesus immunoglobulin (Ig) genes $(19,20)$, examination of $\mathrm{B}$ cell responses in this species is now more accessible.

Phenotypic characterization of PCs in rhesus macaques has primarily focused on the analysis of blood where plasmablasts are abundant 1 week following immunization $(21,22)$. Two recent reports describe phenotypic analysis of BM-resident PCs in macaques based on markers that work well for human BM PCs, including CD138, CD38, CD27, and/or CD19 (23, 24). However, as these markers are also expressed on other cell types and their pattern of expression may be different in macaques, efforts to characterize additional markers that distinguish rhesus macaque BM PCs are needed. Here, we describe a comprehensive phenotypic and functional characterization of rhesus macaque BM PCs. Specifically; we show that antibody-secreting cells (ASC) are contained within the CD20low/-CD138+CD31+ population. This staining panel discriminated BM PCs from peripheral plasmablasts and was also suitable for staining human BM PCs. We show further that the CD19 and CD38 markers, often used to define human BM PCs, are suboptimal for defining rhesus macaque BM PCs due to reduced crossreactivity and/or different expression patterns. Finally, we show that cryopreservation of rhesus BM cells led to a selective loss of the CD20low/-CD138+CD31+ population, which has practical implications for studies of BM PCs.

\section{MATERIALS AND METHODS}

\section{Ethics Statement}

The animal work was conducted with the approval of the regional Ethical Committee on Animal Experiments (Stockholms Norra Djurförsöksetiska Nämnd), while the human work was conducted with the approval of the regional ethical vetting board in Stockholm with the registration number 2015/305-31/1. All methods were carried out in accordance with the approved guidelines.

\section{Animals}

Rhesus macaques (Macaca mulatta) of Chinese origin, 5-6 years old, were housed at the Astrid Fagraeus Laboratory facility at Karolinska Institutet, as previously described $(21,25)$. Housing and care procedures were in compliance with the provisions and general guidelines of the Swedish Board of Agriculture, and the facility has been assigned an Animal Welfare Assurance number by the Office of Laboratory Animal Welfare (OLAW) at the National Institutes of Health (NIH). The macaques were housed in pairs in $4 \mathrm{~m}^{3}$ cages, enriched to give them the possibility to express their physiological and behavioral needs. They were habituated to the housing conditions for more than 6 weeks before the start of the experiment and subjected to positive reinforcement training in order to reduce the stress associated with experimental procedures. All immunizations and blood samplings were performed under sedation with ketamine $10-15 \mathrm{mg} / \mathrm{kg}$ intramuscularly (i.m.) (Ketaminol $100 \mathrm{mg} / \mathrm{ml}$, Intervet, Sweden). BM aspirates were collected as described previously (26). The macaques were weighed at each sampling. All animals were confirmed negative for simian immunodeficiency virus (SIV), simian T-cell lymphotropic virus, simian retrovirus type D, and simian Herpes B virus.

\section{Human Subjects}

Bone marrow cells from three adult human subjects were acquired in conjunction with hip-replacement surgery at the Orthopedics Clinic, Karolinska University Hospital, in Stockholm. Oral and written consent was obtained.

\section{Isolation and Cryopreservation of Mononuclear Cells from Blood and Bone Marrow}

Mononuclear cells from peripheral blood and BM were isolated within $2 \mathrm{~h}$ of sample collection using BD Vacutainer ${ }^{\circledR} \mathrm{K} 2$ EDTA tubes. Tubes were immediately inverted 8-10 times, and blood tubes were centrifuged at $15 \mathrm{~min}$ at $2400 \mathrm{rpm}$ at $25^{\circ} \mathrm{C}$ to separate the plasma. Blood or BM cells were diluted in sterile PBS and layered on top of Ficoll-Paque ${ }^{\mathrm{TN}}$ (GE Healthcare Life Sciences) at a 2:1 ratio, and the tubes were centrifuged at $30 \mathrm{~min}$ at $2200 \mathrm{rpm}$ at $25^{\circ} \mathrm{C}$ without break. A $5 \mathrm{ml}$ sterile serological pipette was used to isolate the buffy coat layer in a $50 \mathrm{ml}$ conical tube. The cells were washed twice with PBS (1750 rpm, $\left.10 \mathrm{~min}, 25^{\circ} \mathrm{C}\right), 3 \mathrm{ml}$ of lysis buffer was added for $5 \mathrm{~min}$, and cells were washed twice with PBS $\left(1500 \mathrm{rpm}, 10 \mathrm{~min}, 25^{\circ} \mathrm{C}\right)$ and resuspended in $5 \mathrm{ml}$ of PBS with 5\% FBS (Sigma) per tube of blood collected. The cells 
were counted using $0.4 \%$ trypan blue exclusion countess (Life Technologies). An aliquot of the cells was used fresh in ELISpot assays, and the remaining cells were frozen at $10-15 \times 10^{6} / \mathrm{ml} /$ cryovial in Fetal Bovine Serum (FBS) with 10\% DMSO chilled at $4^{\circ} \mathrm{C}$. Cryovials containing cells were placed in an ice bath for 15 min prior to placing in a "Mr. Frosty" (Nalgene) cryocontainer before transfer, first to $-80^{\circ} \mathrm{C}$ for $24 \mathrm{~h}$ and then, to liquid nitrogen tanks. To thaw the cells, the cryovials were removed from the liquid nitrogen and brought on dry ice to the lab. Vials were thawed immediately while shaking in a $37^{\circ} \mathrm{C}$ water bath until a few ice crystals remained. No more than one cryovial was thawed at the same time. The cell suspension was transferred into a $10 \mathrm{ml}$ of pre-warmed RPMI 1640 media (Sigma), supplemented 10\% FCS (Sigma), $2 \mathrm{mM}$ L-glutamine, $100 \mathrm{U} / \mathrm{ml}$ penicillin, $100 \mathrm{mM}$ streptomycin, and 2\% HEPES (supplemented media), all from Life Technologies. The tubes were centrifuged at $1500 \mathrm{rpm}$ for 5 min with the brake set at low speed. After one additional wash in supplemented media, the cells were resuspended in $5 \mathrm{ml}$ of PBS. Cells were counted and viability assessed using $0.4 \%$ trypan blue exclusion. The viability typically ranged from 85 to $95 \%$.

\section{Flow Cytometry of BM PCs}

Frozen $\mathrm{BM}$ cells were thawed at $37^{\circ} \mathrm{C}$ and washed twice in prewarmed supplemented media. Cells were washed twice with PBS and counted using 0.4\% trypan blue exclusion countess (Life Technologies), and the cell viability was consistently $>90 \%$. Cells were resuspended in $100 \mu \mathrm{l}$ of PBS in a 96-well plate $\left(1-4 \times 10^{6}\right.$ cells/well $)$ and incubated for $10 \mathrm{~min}$ at $4^{\circ} \mathrm{C}$ with FcR blocking (eBioscience or BD) for rhesus and human cells respectively, and Aqua Dead Cell Stain Kit (Molecular Probes). Cells were washed with FACS buffer (PBS + 2\% FBS). Rhesus cells were surface-stained with antibodies against CD3 clone SP34-2 BV786 (BD), CD20 clone 2H7 BV421 (BD), CD138 clone DL101 PE (eBioscience), CD49d clone 9F10 PerCp-Cy5.5 (eBioscience), CD31 clone WM59 Alexa 647 (BD), CD98 clone 5E5 FITC (eBioscience), CD19 clone J3-119 PE-Cy7 (Beckman Coulter), and CD38 clone OKT10 APC (NIH Non-human primate reagent resource). Human cells were surface-stained using the same Ab panel as used for macaques with the exception of CD38 [clone HIT2 APC (BD)]. All Abs were previously titrated for optimal staining of rhesus macaque and human peripheral blood mononuclear cells (PBMCs). Samples were collected on a FACS LSRII or sorted using a FACS ARIA FUSION (BD Immunocytometry Systems) and analyzed using FlowJo software.

\section{Flow Cytometry of Circulating Plasmablasts in Rhesus Macaque Blood}

Frozen PBMCs were thawed, and cell viability was consistently $>90 \%$. After the incubation with the FcR blocking (BD) and Aqua Dead Cell Stain (Molecular Probes), as described in Section "Flow Cytometry of BM PCs," the cells were washed with FACS buffer and surface-stained for $15 \mathrm{~min}$ at $4^{\circ} \mathrm{C}$ with antibodies against CD3 BV786 (BD), CD20 BV421 (BD), CD138 PE (eBioscience), CD80 clone L307.4 APC-H7 (BD), HLA-DR clone L243 PerCP-Cy5.5 (BD), CD14 clone M5E2 BV786 (BD), CD16 clone 3G8 BV786 (BD), CD98 FITC (eBioscience), and CD49d
APC (BD) or CD31 Alexa 647 (BD). Cells were washed twice with FACS buffer and incubated in $100 \mu \mathrm{l}$ of Cytofix/Cytoperm (BD) for $20 \mathrm{~min}$ at $4^{\circ} \mathrm{C}$. Cells were then washed twice in Perm/ Wash buffer (BD) and intracellularly stained in $100 \mu \mathrm{l}$ of Perm/ Wash buffer for $15 \mathrm{~min}$ at $4^{\circ} \mathrm{C}$ with IgG clone G18-145 PE-Cy7 (BD), followed by two washes in Perm/Wash buffer and two in FACS buffer. All Abs were previously titrated for optimal staining of rhesus macaque PBMCs. Samples were collected on a FACS LSRII (BD Immunocytometry Systems) and analyzed using FlowJo software.

\section{B Cell ELISpot Assay}

Plasma cells were enumerated as previously described (21). In brief, to evaluate the presence of BM PCs, defined as spontaneous ASC, different gate populations were sorted into supplemented media and plated directly into ELISpot plates. To detect ASC, MAIPSWU1096-well plates (Millipore) were coated with $10 \mu \mathrm{g} / \mathrm{ml}$ antihuman IgG, IgA, and IgM (Fc $\gamma, F c \alpha$, and Fc $\mu$ chain) (Jackson ImmunoResearch), and cells were transferred to the plates in dilution series and incubated for $18 \mathrm{~h}$ at $37^{\circ} \mathrm{C}, 5 \% \mathrm{CO}_{2}$. The plates were then washed with PBS containing $0.05 \%$ Tween and incubated with biotinylated antihuman IgG, IgA, or IgM $(0.25 \mu \mathrm{g} /$ $\mathrm{ml}$ ) for $1.5 \mathrm{~h}$ at $37^{\circ} \mathrm{C}$ followed by washing and incubation with streptavidin-AP (1:1000, Mabtech). The reactions were developed using BCIP/NBT substrate (Sigma) and stopped by washing in distilled water. Spots corresponding to ASC were counted using an ImmunoSpot ${ }^{\circledR}$ analyzer (Cellular Technology Ltd.). The results were converted to show percentage of ASC of plated events.

\section{Transmission Electron Microscopy}

CD138+CD31+ cells were sorted from a frozen sample, and 34,000 events were acquired into supplemented media. The cells were washed twice with PBS before fixing in $2.5 \%$ glutaraldehyde in $0.1 \mathrm{M}$ phosphate buffer, $\mathrm{pH} 7.4$ at $+4^{\circ} \mathrm{C}$ overnight, and centrifuged to a pellet. The pellet was rinsed in $0.1 \mathrm{M}$ phosphate buffer, pH 7.4. A small amount of $10 \%$ gelatine was added on top of the small pellet and left to solidify. The pellet was then fixed in $2 \%$ osmium tetroxide in $0.1 \mathrm{M}$ phosphate buffer, $\mathrm{pH} 7.4$ at $+4^{\circ} \mathrm{C}$ for $2 \mathrm{~h}$, dehydrated in ethanol followed by acetone and embedded in LX-112 (Ladd, Burlington, VT, USA). Ultrathin sections $(\sim 50-60 \mathrm{~nm})$ were cut using a Leica EM UC 6 (Leica, Wien, Austria). Sections were contrasted with uranyl acetate followed by lead citrate and visualized in a Hitachi HT 7700 (Tokyo, Japan) at $80 \mathrm{kV}$. Digital images were taken by using a Veleta camera (Olympus Soft Imaging Solutions, GmbH, Münster, Germany).

\section{Statistical Analysis}

All statistical analysis was done with GraphPad Prism software version 6 and considered significant at ${ }^{*} p \leq 0.05$, ${ }^{* *} p \leq 0.01$, ${ }^{* * *} p \leq 0.001$, and ${ }^{* * *} p \leq 0.0001$. Comparisons of $\geq 3$ groups were analyzed by the ANOVA non-parametric Kruskal-Wallis test followed by Dunn's post test for individual comparisons. For repetitive measures, ANOVA was used. When comparing two groups, $t$-test or Mann-Whitney test were used. To analyze groups with multiple factors, two-way ANOVA was used followed 
by Bonferroni's post test for multiple comparisons. The relationship between variables was established with the non-parametric Spearman's correlation analysis.

\section{RESULTS}

\section{CD3-CD20low/-CD138+ Bone Marrow Cells in Rhesus Macaques Are Enriched for IgG-Secreting Cells}

Bone marrow-resident PCs in humans are phenotypically defined as CD3 negative cells that have downregulated their surface expression of CD20 and express high levels of CD38 and CD138. Functionally, the hallmark of PCs is their capacity to secrete Ab constitutively both in vivo and when cultured in vitro. In rhesus macaques, which closely mimic humans both genetically and in regards to immune cell phenotypes, BM PCs were defined as CD20-CD19+CD38+CD138+ cells $(23,24)$. While this provides a useful starting point, it was shown that the CD19+CD20population in cynomolgus macaques contained rhesus B-1-like B cells (27), and in humans, both CD19+ and CD19- populations harbor BM PCs $(16,17)$. Furthermore, currently available CD19 antibodies stain rhesus CD19 suboptimally (Figure S1 in Supplementary Material); thus, the definition of additional markers of rhesus BM PCs coupled with functional analysis is needed for improved definition of these cells.

To meet this objective, we first examined if CD3-CD20low/CD138+ BM cells could be functionally defined as Ab-secreting PCs $(23,24)$. We stained Ficoll-separated BM cells from nine rhesus macaques for CD3, CD20, and CD138. The analysis of one representative animal is shown as dot plots (Figure 1A), and data from all nine macaques are shown as mean values (Figure 1B). The mean frequency of CD3-CD20+ cells of live cells was $15 \pm 7 \%$, while the mean frequency of CD3-CD20low/- cells in the live gate was $85 \pm 7 \%$. To stain for CD138, we used the DL101 Ab clone, which previously was demonstrated to be crossreactive with rhesus macaque CD138 (22-24). Staining of the LiveCD3-CD20low/- population resulted in distinct CD138and CD138+ populations with $1.62 \%$ of the LiveCD3-CD20low/cells being CD138+ in the representative animal (Figure 1A), and the mean frequency of CD138+ cells of the LiveCD3- cells for the nine macaques was $1.8 \pm 0.8 \%$ (Figure $1 \mathbf{B}$ ). We next wished to couple phenotype to function and sorted three populations of cells, LiveCD3-CD20+ cells, LiveCD3-CD20low/-CD138cells, and LiveCD3-CD20low/-CD138+ cells, from three macaques. The cells were sorted directly into ELISpot plates coated with anti-IgG $\mathrm{Ab}$ and incubated for $18 \mathrm{~h}$ without additional stimulation to assess their capacity to constitutively produce IgG. IgG-secreting PCs were observed in the CD3-CD20low/-CD138+ population $(8 \pm 4 \%)$, while virtually no IgG ASC were observed in the other two populations $\mathrm{CD} 3-\mathrm{CD} 20+(0.08 \pm 0.1 \%)$ and CD3-CD20low/-CD138$(0.03 \pm 0.06 \%)$, respectively (Figure $1 \mathrm{C})$. Considering that only $8 \%$ of CD3-CD20low/-CD138+ BM cells produced IgG, it appeared likely that a large proportion of the CD138+ cells were not functional PCs. Therefore, we examined the CD138+ cells for additional markers that would allow for an improved enrichment of Ab-secreting BM cells.

\section{IgG-, IgA-, and IgM-Secreting BM PCs Are Contained Within the CD138+CD31+ Population}

To identify additional markers that could enrich rhesus macaque BM PCs, we selected three molecules that were previously reported to be expressed by human or mouse PCs: CD49d, shown to be highly expressed on human BM PCs $(15,28)$, CD31, reported to be expressed on PCs in human spleen and
A

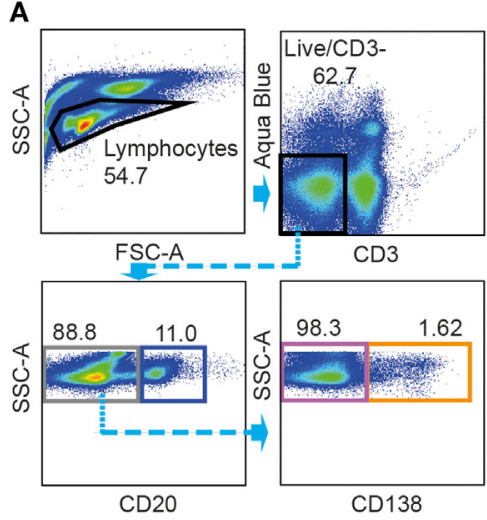

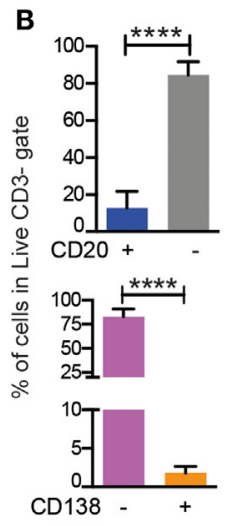

C

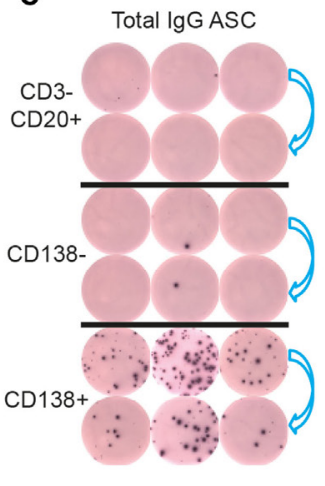



FIGURE 1 | Rhesus BM IgG ASC are found in the CD20low/-CD3-CD138+ population. (A) FACS gating strategies for CD20+ (blue), CD20- (gray) populations gated from live, CD3- cells, and for CD138+ (orange) and CD138- (purple) populations gated from live, CD3-CD20low/- rhesus cells. (B) Frequency (mean and SD) of rhesus CD20+, CD20-, and CD138+, CD138- cells from the live, CD3-. Mann-Whitney test was done ${ }^{\star \star \star \star} p<0.0001(n=9)$. (C) ELISpot for total IgG ASC from the sorted populations shown in (A) are CD138+ (orange sort gate), CD138- (purple sort gate), and CD3-CD20+ (gray sort gate). Data from three different animals are shown, and the sorted cells were plated in two different dilutions (left panel). Frequency (mean and SD) of total lgG ASC in the three sorted populations are labeled as: CD138+, CD138-, and CD3-CD20+. Kruskal-Wallis test followed by Dunn's multiple comparison were performed * $p<0.05$ and ${ }^{\star \star} p<0.01(n=5)$. All data were obtained from frozen samples. 
BM (29), and CD98, which was recently suggested to be a marker of mouse PCs (30). We stained rhesus macaque CD138+ BM cells for CD31, CD49d, and CD98 and found that all three markers co-stained a subset of the CD138+ cells (Figure 2A). There was no statistically significant difference between the $\mathrm{CD} 138+\mathrm{CD} 31+(0.95 \pm 0.4 \%), \mathrm{CD} 138+\mathrm{CD} 49 \mathrm{~d}+(0.8 \pm 0.4 \%)$, and CD138+CD98+ $(0.7 \pm 0.4 \%)$ populations. We observed a high correlation in the frequencies between the CD138+CD31+ population, the CD138+CD49d+ $\left(r=0.9,{ }^{* *} p<0.01\right)$, and the CD138+CD98+ $\left(r=0.8,{ }^{* *} p<0.01\right)$ population (Figure $2 B$ ), which suggests that CD31, CD49d, and CD98 stained the same population. Therefore, we focused on one of the markers, CD31, for the subsequent analysis.

To determine which population harbored Ab-secreting PCs, we next sorted LiveCD3-CD20low/-CD138+CD31+ and Liv eCD3-CD20low/-CD138+CD31- cells from fresh rhesus BM into anti-IgG-, anti-IgA-, or anti-IgM-coated ELISpot plates. LiveCD3-CD20low/-CD138- cells were included as a negative control (Figure 3A, upper panel). Only the double-positive
CD138+CD31+ population was significantly enriched for ASC (Figure 3A, lower panel). Combining the results obtained from 12 animals, we found that $24 \%$ of the plated CD $138+C D 31+$ cells produced antibodies with the majority being of IgG isotype $(14.5 \pm 6.0 \%)$, followed by $\operatorname{IgA}(7.9 \pm 3.7 \%)$ and $\operatorname{IgM}(1.8 \pm 1.2 \%)$ (Figure 3B). These findings are consistent with studies of human BMPCs (16). When the plated CD138+CD31- cells were similarly analyzed, we found that $3 \%$ of the plated cells secreted antibodies with no significant difference between the IgG- $(1.6 \pm 2.5 \%)$, IgA$(1.1 \pm 2.2 \%)$, and IgM-secreting $(0.2 \pm 0.2 \%)$ cells (Figure $3 \mathrm{~B})$. To assess $\mathrm{PC}$ enrichment in the sorted CD138+CD31+ population by morphological analysis, we performed Transmission Electron Microscopy (TEM). We found that PCs were the predominant cell type in these specimens, as distinguished by characteristic PC morphology, including nuclei with "spokes of a wheel" chromatin structure and prominent rough ER in the majority of the cells (Figure 3C). Altogether, these results illustrate that the $\mathrm{CD} 138+\mathrm{CD} 31+$ rhesus BM population comprises the functional IgG-, IgA-, and IgM-secreting PC compartment.
A

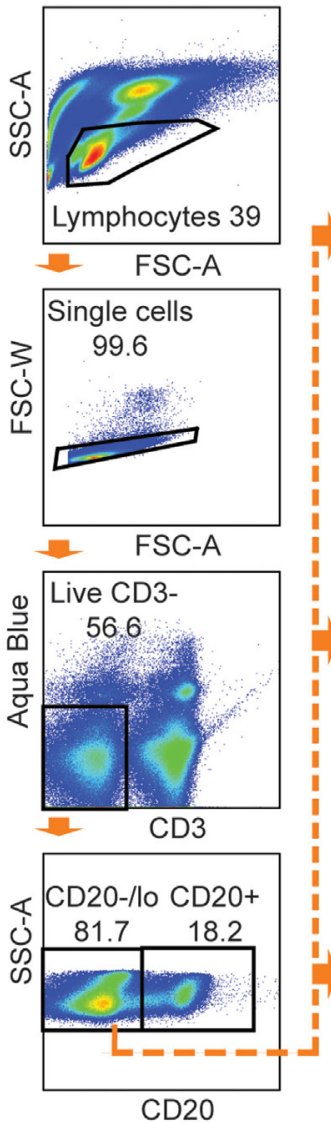

B
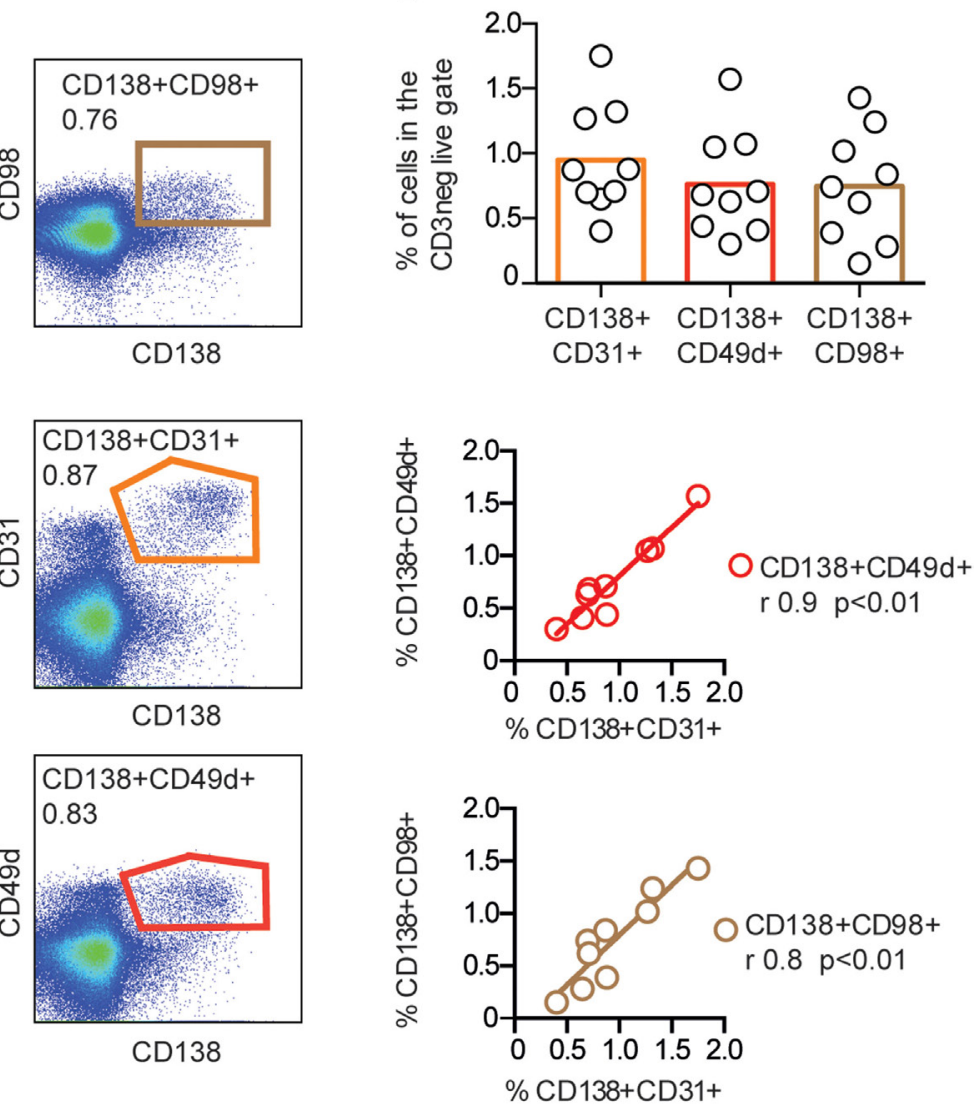

FIGURE 2 | CD31, CD49d, and CD98 expression of CD138+ rhesus bone marrow cells. (A) Representative FACS plots showing CD138+CD31+, CD138+CD49d+, and CD138+CD98+ populations gated from live, CD3-CD20low/- cells in the same stain of rhesus bone marrow. (B) Frequency (mean) of CD138+CD31+, CD138+CD49d+, and CD138+CD98+ populations (upper panel). Correlations between CD138+CD31+, CD138+CD49d+, and CD138+CD98+ populations were analyzed using non-parametric Spearman $(r)\left({ }^{* *} p<0.01\right)$ and linear regression (middle and lower panels) $n=9$. All data were obtained from frozen samples. 


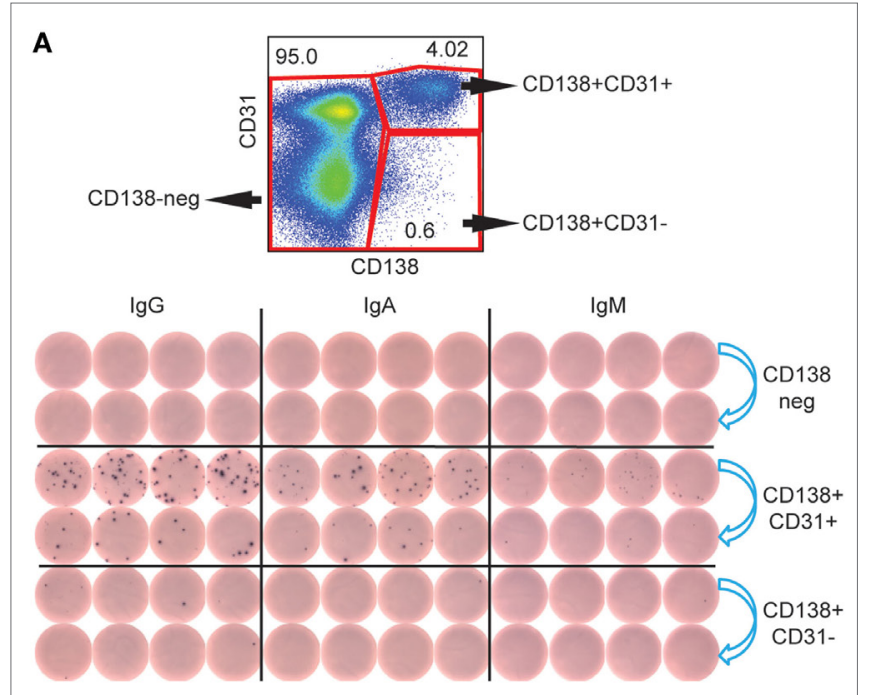

B

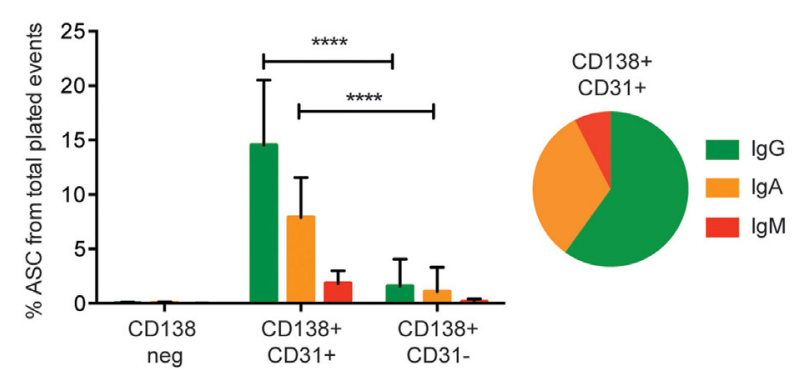

C

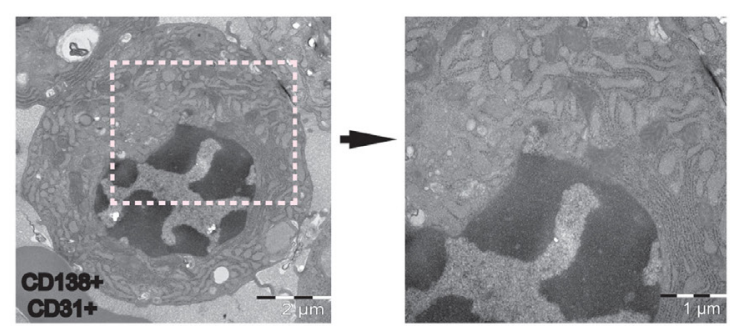

FIGURE 3 | IgG-, IgA- and IgM-secreting PCs are found in the CD138+CD31+ population. (A) Representative FACS plot showing the gates of the three sorted populations (red gates), CD138+CD31+, CD138+CD31-, and CD138- (upper panel). Representative B cell ELISpot data of three experiments showing the sorted populations tested for lgG, IgA, and IgM secretion. 2000 events were sorted from each population, 2 dilutions of cells were plated at fourfold dilutions (blue arrows) (lower panel). (B) Frequencies (mean and SD) of IgG (green), IgA (orange), and $\mathrm{IgM}$ (red) ASC of total plated events from the three sorted populations. Pie chart of the CD138+CD31+ population, $60 \%$ of the cells secreted IgG (green), $32.5 \% \operatorname{lgA}$ (orange), and $7.5 \% \operatorname{lgM}$ (red). Data were obtained from fresh rhesus BM $(n=12)$. Two-way ANOVA followed by Tukey's multiple comparison test was performed, ${ }^{* \star * *} p<0.0001$. (C) TEM analysis of cells sorted from the CD138+CD31+ population, exhibiting characteristic plasma cell morphology with typical "spokes of a wheel" chromatin structures, and prominent and dilated RER. Two different magnifications are shown with scale bars of $2 \mu \mathrm{m}$ (left panel) and $1 \mu \mathrm{m}$ (right panel).

$(\mathbf{A}, \mathbf{B})$ data were obtained from fresh samples. (C) data were obtained from frozen samples.

\section{Co-expression of CD138, CD31, CD49d, and CD98 Distinguishes BM Plasma Cells from Peripheral Plasma Cells}

We next sought to examine if the expression pattern of CD138, CD31, CD49d, and CD98 on blood PCs was similar to the one found in BM PCs. Circulating rhesus plasmablasts peaking 1 week after immunization were recently defined as CD3-CD 16-CD20-/intHLA-DR+CD 14-CD 11c-CD 123 $-\mathrm{CD} 80+$ cells (22). We used the same staining panel with the exception of CD11c and CD123 (Figure S2A in Supplementary Material). To determine the percentage of these cells that costained with IC IgG, we examined samples collected 7 days after vaccination from a previous study (21). We found that $47 \pm 14 \%$ of the LiveCD3-CD16-CD14-CD20-/intHLA$\mathrm{DR}+\mathrm{CD} 80+$ were positive for IC IgG (Figure 4A). Thus,
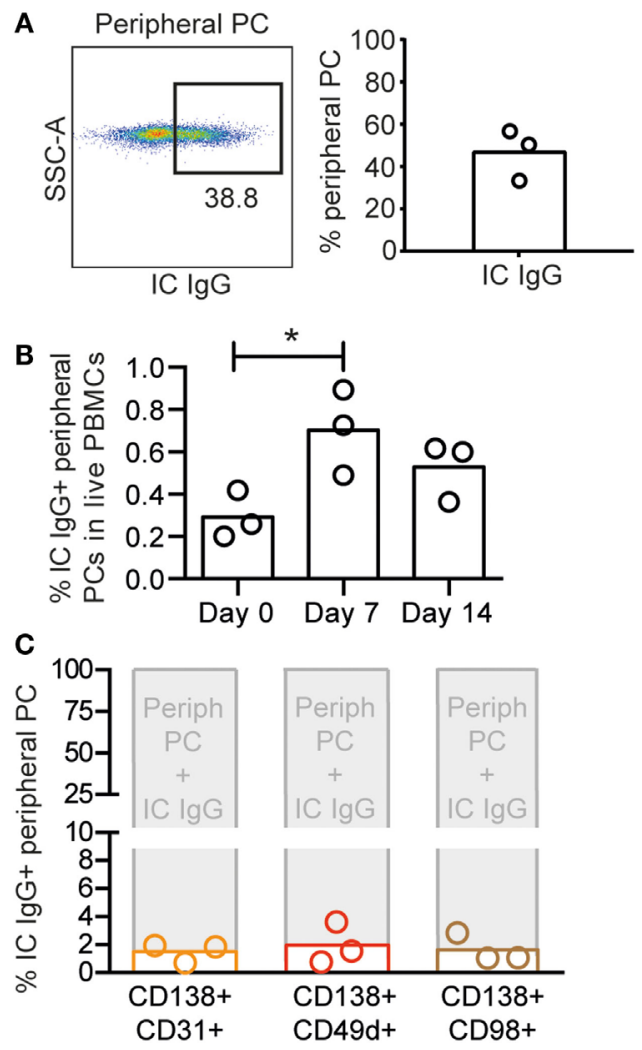

FIGURE 4 | Circulating plasmablasts in rhesus macaques are phenotypically distinct from BM PCs. (A) Representative intracellular (IC) IgG plot (left panel) of rhesus peripheral PCs and percentages IC IgG+ cells of CD14-CD16-CD20low/-HLA-DR+CD80+ cells from day 7

postimmunization $(n=3$ ) (right panel). (B) Frequency of IC IgG+ peripheral PCs in rhesus PBMCs collected on days 0,7 , and 14 postimmunization. To determine statistical significance, ${ }^{*} p<0.05$, ANOVA was used for repetitive measures followed by Holm-Sidak's multiple comparison (C) Percentages of CD138+CD31+ (orange), CD138+CD49d+ (red), and CD138+CD98+ (brown) populations (defined in Figure S2B in Supplementary Material) in IC IgG+ peripheral PCs. Each bar represents the mean, and each circle represents one animal $(n=3)$. All data were obtained from frozen samples. 
plasmablasts were defined as LiveCD3-CD16-CD14-CD20-/ intHLA-DR+CD80+IC IgG+ cells. Analysis of PBMCs collected 0,7 , or 14 days after vaccine inoculation (21) corroborated the occurrence of the peak peripheral PC response 7 days after immunization $\left({ }^{*} p<0.05\right.$, ANOVA for repetitive measures) (Figure 4B). We next examined if CD14-CD16-CD20low/-HLA-DR+CD80 + IC IgG+ cells from the 7-day time point were CD138+CD31+, CD138+CD49d+ or CD138+CD98 and found negligible coexpression (Figure 4C) but rather single expression of CD31, CD49d, and CD98 due to the absence of CD138 expression on peripheral PCs (Figure S2B in Supplementary Material), in stark contrast to the phenotype of BM-resident PCs that display high expression of both CD138 and CD31.

\section{Human and Macaque BM PCs Similarly Co-express CD138 and CD31}

Having established that rhesus BM PCs are contained within the CD138+CD31+ population, we performed a direct comparison between macaque and human $\mathrm{BM}$ to determine if the same staining panel was suitable for identification of human BM PCs. The purpose of this was to perform a qualitative rather than quantitative comparison of PC populations in these hosts as the number of available human subjects was low. As mentioned earlier, CD38 and CD19 are used to define different subsets of human BM PCs. Hence, antibodies against these markers were added to the rhesus PC-staining panel, and BM samples from three human donors and three rhesus macaques were examined (Figures 5A,B). We clearly distinguished a CD138+CD31+ human BM PC population that also expressed high levels of CD38, while the same staining applied to rhesus macaque BM PCs gave only a dim CD38 signal (Figure 5C). Furthermore, CD38 expression robustly separated the human CD138+CD31+ and CD138+CD31- populations, while this was not the case for the corresponding rhesus BM cells (Figure 5D).

The distribution of CD138+CD31+ and CD138+CD31- populations was similar in rhesus and human BM samples. However, while CD138+CD31+ cells were positive for CD38 in both humans $(95.6 \pm 2.3 \%$ of cells defined as CD38++) and macaques $(91.5 \pm 2.8 \%$ of cells defined as CD38+) (Figure $5 \mathrm{E}$, left), the CD138+CD31- population (Figure 5E, right) was negative for CD38 in humans $(2.3 \pm 0.5 \%)$, while a significant proportion of the rhesus macaque CD138+CD31- cells $\left(63.9 \pm 8.9 \%,{ }^{* *} p<0.01\right)$ expressed CD38 to a similar intensity as the CD138+CD31+ population. Additionally, we evaluated CD19 expression in human $\mathrm{CD} 138+\mathrm{CD} 31+\mathrm{CD} 38++$ population and in rhesus CD138+CD31+CD38+ population, and we found that both CD19+ and CD19- populations were clearly distinguishable on human cells but less so on rhesus cells (Figures 5A,B). Thus, the CD3-CD20low/-CD138+CD31+ staining panel identifies PCs in both human and rhesus BM, while markers frequently used to define human PCs, CD38 and CD19, are suboptimal for staining rhesus macaque BM PCs.
A

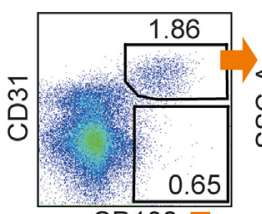
CD138

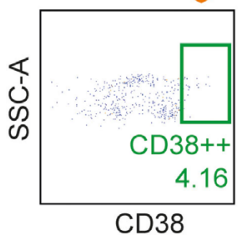

C

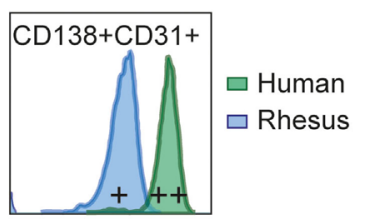

CD38
B

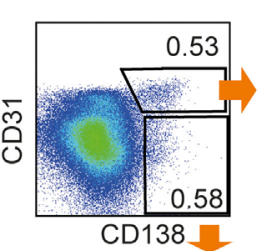

CD38

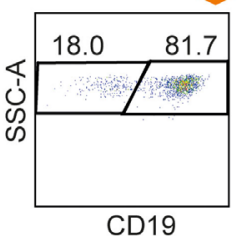

D
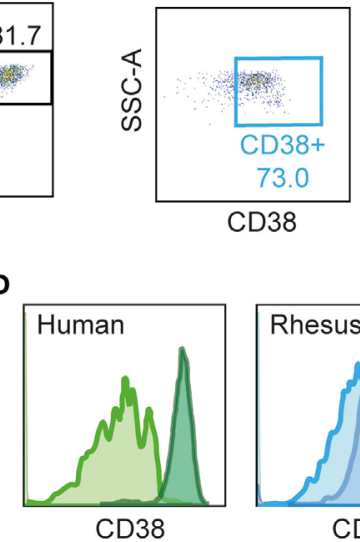

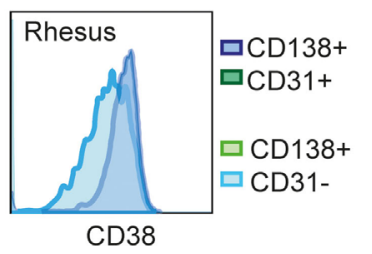

E


Human $\square$ Rhesus
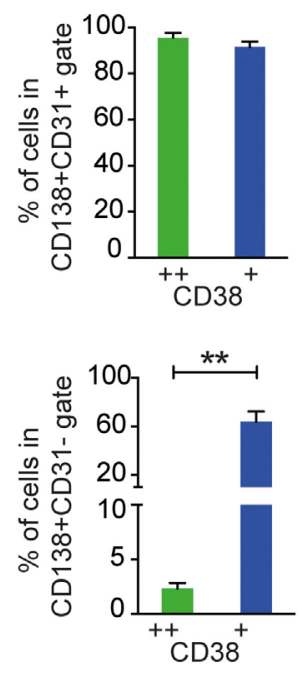

FIGURE 5 | Examination of CD19 and CD38 expression on human and rhesus CD3-CD20low/-CD138+CD31+ BM cells. Flow cytometric analysis of (A) human BM and (B) rhesus BM using the CD138+CD31+ gating strategy with the addition of CD38 and CD19. CD138+CD31+ cells were gated from live CD3-CD20low/-. (C) Representative histogram of CD38 expression showing that CD138+CD31+ cells co-expressed CD38 in both human and rhesus BM, but with lower staining intensity in rhesus BM. (D) Representative histograms of the CD38 marker showing the separation of CD138+CD31+ from the CD138+CD31- population in human cells (dark and light green, respectively) and in rhesus cells (dark and light blue, respectively). (E) Percentages of CD38+ cells within the CD138+CD31+ population (top panel) and CD138+CD31 - population (bottom panel) for human cells (green) and rhesus cells (blue). Unpaired $t$-tests were done using Welch's correction to determine statistical significance, ${ }^{\star \star} p<0.01$. Bars and error bars represent mean and SD, respectively $(n=3)$. All data were obtained from frozen samples. 


\section{Cryopreservation of Macaque Bone Marrow Cells Results in a Selective Loss of CD138+CD31+ Cells}

Rhesus macaques are widely used to evaluate immune responses induced by vaccine candidates under development for human clinical use. In most of these immunization studies, samples are collected and immediately frozen for subsequent analysis. We observed a striking difference in the frequency of $\mathrm{CD} 138+\mathrm{CD} 31+$ cells between fresh and frozen rhesus macaque $\mathrm{BM}$ cells (Figure 6). When staining fresh and frozen BM from a representative animal using the same gating, the frequency of CD138+CD31+ population in the LiveCD3-CD20low/- gate was 10 times higher in the fresh sample (4.73\%) than in the frozen sample $(0.42 \%)$ (Figure 6A). When extending our analysis to BM samples from 12 rhesus macaques, we observed a significantly higher frequency of CD138+CD31+ cells in fresh samples (4.2 \pm $5.3 \%)$ than in the frozen samples $(0.8 \pm 0.4 \%)$ (Figures 6B,C) $\left({ }^{*} p<0.05\right.$, Dunn's multiple comparison test). The frequency of CD138+CD31- cells was similar in fresh and frozen samples indicating a selective loss of CD138+CD31+ PCs following freezing (Figures 6B,C). The selective loss of CD138+CD31+ after the cryopreservation highlights the advantage of working with fresh BM samples, which is relevant for studies that aim to analyze Ab-secreting PCs in this compartment.

\section{DISCUSSION}

The induction of persistent $\mathrm{Ab}$ responses is the goal of most prophylactic vaccines. Although rhesus macaques are frequently used for preclinical evaluation of human vaccine candidates, a comprehensive analysis of the phenotypic and functional properties of rhesus macaque BM PCs is lacking. Here, we demonstrate that IgG-, IgA-, and IgM-secreting rhesus BM PCs were contained within the CD31+ population of the CD20low/-CD138+ cells.

CD31 stains both BM PCs and periphery PCs; however, the CD20low/-CD138+CD31+ panel was specific for BM PCs as it did not stain peak (7 days after a booster immunization) peripheral PCs, which are CD138-. Human BM PCs are either CD19+ or CD19-, with recent reports demonstrating that long-lived PCs are contained in the CD19- compartment $(16,17)$. In this regard, we note that currently available anti-CD19 Abs, including clone J3-119, resolve rhesus CD19+ and CD19- populations suboptimally. Therefore, we opted to exclude this marker from our panels, except in the experiments where direct comparisons with human $\mathrm{BM}$ were performed. In those experiments, we demonstrate that unlike on human cells, both CD20low/-CD138+CD31+ and CD20low/-CD138+CD31- rhesus BM cells stained positive for CD38, suggesting that this marker is also not suitable to define rhesus BM PC.

In addition to CD31, we evaluated the adhesion molecule CD49d and the lymphocyte activation antigen CD98 as markers for rhesus BM PCs. These markers were previously shown to be expressed on human or mouse PCs; CD138, CD31, and CD49d are involved in PCs interactions with the BM microenvironment, and their overexpression is a key feature of healthy human BM PCs $(15,29,31)$. CD31 is expressed on a large number of cell types, and it has been used to isolate human PCs from BM $(32,33)$, tonsils (34), lamina propria (31), and spleen (29), belongs to the Ig gene superfamily, and is the ligand of CD38
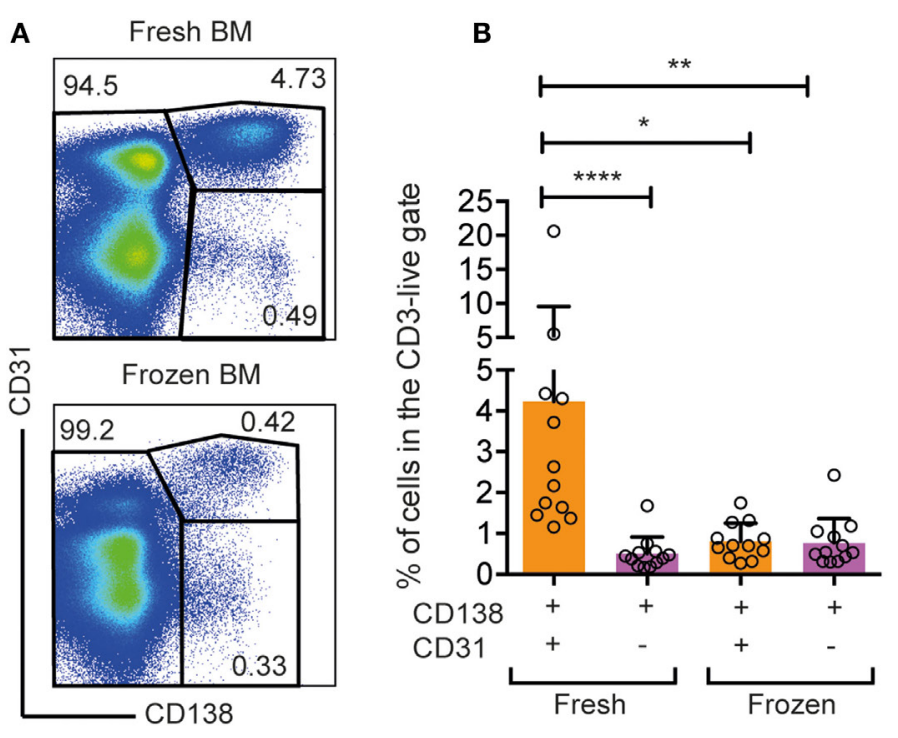

C

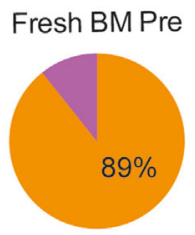

Frozen BM Pre

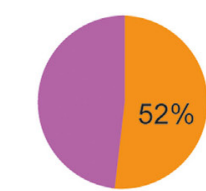

CD138+CD31+

CD138+CD31-

FIGURE 6 | Selective loss of CD138+CD31+ cells in frozen rhesus bone marrow samples. (A) Representative flow cytometry dot plots of CD138+CD31+, CD138+CD31-, and CD138- populations gated from live, CD3-CD20low/- cells isolated from fresh and frozen bone marrow. (B) Frequencies of CD138+CD31+ (orange) and CD138+CD31- (purple) cells gated from live, single CD3-CD20low/-cells. Kruskal-Wallis test followed by Dunn's multiple comparison test for non-parametric data was performed $(n=12)$. Significant differences were determined as ${ }^{\star} p<0.05,{ }^{\star \star} p<0.01$, and ${ }^{\star \star \star \star} p<0.0001$. (C) Pie charts showing percentages of CD138+CD31+ (orange) and CD138+CD31- (purple) populations in frozen and fresh samples. 
(35). CD49d is a transmembrane protein, which associates with integrin beta 1 to form the heterodimer VLA-4 and is a prognostic marker for chronic lymphocytic leukemia (CLL) $(36,37)$. CD98 is important for activation, proliferation, and effector functions of T and B cells $(38,39)$ and was recently described as a marker for BM PCs in mice (30). We demonstrate here that CD31 expression correlates strongly with CD49d and CD98 expression on CD20low/-CD138+ rhesus BM cells.

In humans, the distribution of PC-produced Ig isotypes varies in different organs. For instance, IgG predominates in the BM followed by $\operatorname{IgA}(16,40)$, while in the spleen, the distribution is more equal (29), and IgA is most abundant in mucosa-associated lymphoid tissues (41). In our studies, rhesus BM PCs produced primarily $\operatorname{IgG}(60 \%)$, followed by $\operatorname{IgA}(33 \%)(16,40)$. The presence of IgA-secreting cells in the BM of rhesus and humans is interesting and represents an important area of investigation to complement recent reports of PCs in the gut $(40,42,43)$. The relationship between $\mathrm{Ab}$ specificities encoded by antigen-specific memory B cells, BM PCs, and circulating Abs is of significant interest for our understanding of vaccine-induced responses. Data from the mouse model suggest that the peripheral memory $\mathrm{B}$ cell compartment retains a larger diversity of Ab specificities against a given antigen than does the BM PC compartment (44, 45). In humans, studies have compared the specificities of antigen-specific BM PCs with serum Abs in individuals vaccinated with Tetanus toxoid (46) or infected with HIV-1 (47), but so far rhesus macaques were not used to address such questions despite their frequent use in vaccine evaluation. We also demonstrate a selective loss of CD138+CD31+ cells upon cryopreservation of BM cells using the conditions described in Section "Materials and Methods." Similarly, others have reported a reduction in the frequency of plasmablasts following cryopreservation of PBMCs, as shown by both by flow cytometry and B cell ELISpot analyses (48). These observations have important implications for studies of the $\mathrm{Ab}$ repertoires in these compartments.

In conclusion, we show that functional rhesus macaque BM PCs secreting IgG, IgA, and IgM are highly enriched in the CD20low/-CD138+CD31+ population. These studies offer a foundation for studies aimed at investigating the quality of

\section{REFERENCES}

1. De Silva NS, Klein U. Dynamics of B cells in germinal centres. Nat Rev Immunol (2015) 15:137-48. doi:10.1038/nri3804

2. Tellier J, Shi W, Minnich M, Liao Y, Crawford S, Smyth GK, et al. Blimp-1 controls plasma cell function through the regulation of immunoglobulin secretion and the unfolded protein response. Nat Immunol (2016) 17:323-30. doi:10.1038/ni.3348

3. Moser K, Tokoyoda K, Radbruch A, Maclennan I, Manz RA. Stromal niches, plasma cell differentiation and survival. Curr Opin Immunol (2006) 18:265-70. doi:10.1016/j.coi.2006.03.004

4. Fairfax KA, Kallies A, Nutt SL, Tarlinton DM. Plasma cell development: from B-cell subsets to long-term survival niches. Semin Immunol (2008) 20:49-58. doi:10.1016/j.smim.2007.12.002

5. Tokoyoda K, Egawa T, Sugiyama T, Choi BI, Nagasawa T. Cellular niches controlling B lymphocyte behavior within bone marrow during development. Immunity (2004) 20(6):707-18. doi:10.1016/j.immuni.2004.05.001

6. Radbruch A, Muehlinghaus G, Luger EO, Inamine A, Smith KG, Dörner $\mathrm{T}$, et al. Competence and competition: the challenge of becoming a long-lived $\mathrm{Ab}$ responses in an important primate model and an approach for isolating single PCs from the rhesus macaque BM compartment for $\mathrm{Ab}$ cloning and transcriptional analyses.

\section{AUTHOR CONTRIBUTIONS}

PM-M designed, performed, and analyzed the experiments shown in Figures 1, 2, 3, and 6; LP designed, performed, and analyzed the experiments shown in Figures 4 and 5; CS contributed valuable inputs on the results and the manuscript; $\mathrm{KH}$ performed the transmission electron microscopy; MS provided the rhesus macaque bone marrow samples; PW provided human bone marrow samples; $\mathrm{GH}$ designed the experiments, reviewed the results, and wrote the paper together with PM-M.

\section{ACKNOWLEDGMENTS}

We thank the personnel at Astrid Fagraeus laboratory at Karolinska Institutet for expert assistance with rhesus macaques. We also thank Kiran Sedimbi for assistance in the flow cytometry facility of the Department of Microbiology, Tumor and Cell Biology and Monika Àdori for providing helpful comments on the manuscript. Reagents used in these studies were provided by the NIH Non-human Primate Reagent Resource (R24 RR016001) and NIAID contract (HHSN 2722000900037C).

\section{FUNDING}

This work was funded by a Karolinska Institutet KID grant to PM-M, a medical internship combined with research (Forskar-AT) grant to LP, a Swedish Research Council international postdoc fellowship to CS, an NIH P01 grant AI104722 (HIVRAD) to GKH, and an International AIDS Initiative (IAVI) grant to $\mathrm{GKH}$.

\section{SUPPLEMENTARY MATERIAL}

The Supplementary Material for this article can be found online at http://journal.frontiersin.org/article/10.3389/fimmu.2016.00242

long-lived plasma cell. Nat Rev Immunol (2006) 6:741-50. doi:10.1038/ nri1886

7. Nutt SL, Hodgkin PD, Tarlinton DM, Corcoran LM. The generation of antibody-secreting plasma cells. Nat Rev Immunol (2015) 15:160-71. doi:10.1038/ nri3795

8. Winter O, Mohr E, Manz RA. Alternative cell types form a multi-component-plasma-cell-niche. Immunol Lett (2011) 141:145-6. doi:10.1016/j. imlet.2011.07.006

9. Winter O, Moser K, Mohr E, Zotos D, Kaminski H, Szyska M, et al. Megakaryocytes constitute a functional component of a plasma cell niche in the bone marrow. Blood (2010) 116:1867-75. doi:10.1182/blood-2009-12259457

10. Chu VT, Frohlich A, Steinhauser G, Scheel T, Roch T, Fillatreau S, et al. Eosinophils are required for the maintenance of plasma cells in the bone marrow. Nat Immunol (2011) 12:151-9. doi:10.1038/ni.1981

11. Tangye SG. Staying alive: regulation of plasma cell survival. Trends Immunol (2011) 32:595-602. doi:10.1016/j.it.2011.09.001

12. Kometani K, Kurosaki T. Differentiation and maintenance of long-lived plasma cells. Curr Opin Immunol (2015) 33:64-9. doi:10.1016/j.coi.2015.01.017 
13. Peperzak V, Vikström I, Walker J, Glaser SP, Lepage M, Coquery CM, et al. Mcl-1 is essential for the survival of plasma cells. Nat Immunol (2013) 14:290-7. doi:10.1038/ni.2527

14. Klein U. Programming plasma cell survival. JExp Med (2014) 211:744. doi:10.1084/jem.2115insight1

15. Medina F, Segundo C, Campos-Caro A, Gonzalez-Garcia I, Brieva JA. The heterogeneity shown by human plasma cells from tonsil, blood, and bone marrow reveals graded stages of increasing maturity, but local profiles of adhesion molecule expression. Blood (2002) 99:2154-61. doi:10.1182/blood.V99.6.2154

16. Mei HE, Wirries I, Frolich D, Brisslert M, Giesecke C, Grun JR, et al. A unique population of IgG-expressing plasma cells lacking CD19 is enriched in human bone marrow. Blood (2015) 125:1739-48. doi:10.1182/blood-2014-02-555169

17. Halliley JL, Tipton CM, Liesveld J, Rosenberg AF, Darce J, Gregoretti IV, et al. Long-lived plasma cells are contained within the CD19(-)CD38(hi)CD138(+) subset in human bone marrow. Immunity (2015) 43:132-45. doi:10.1016/j. immuni.2015.06.016

18. Gibbs RA, Rogers J, Katze MG, Bumgarner R, Weinstock GM, Mardis ER, et al. Evolutionary and biomedical insights from the rhesus macaque genome. Science (2007) 316:222-34. doi:10.1126/science.1139247

19. Sundling C, Li Y, Huynh N, Poulsen C, Wilson R, O’dell S, et al. Highresolution definition of vaccine-elicited $\mathrm{B}$ cell responses against the HIV primary receptor binding site. Sci Transl Med (2012) 4:142ra196. doi:10.1126/ scitranslmed. 3003752

20. Sundling C, Phad G, Douagi I, Navis M, Karlsson Hedestam GB. Isolation of antibody $\mathrm{V}(\mathrm{D}) \mathrm{J}$ sequences from single cell sorted rhesus macaque $\mathrm{B}$ cells. J Immunol Methods (2012) 386:85-93. doi:10.1016/j.jim.2012.09.003

21. Sundling C, Forsell MN, O’dell S, Feng Y, Chakrabarti B, Rao SS, et al. Soluble HIV-1 Env trimers in adjuvant elicit potent and diverse functional B cell responses in primates. J Exp Med (2010) 207:2003-17. doi:10.1084/ jem. 20100025

22. Silveira EL, Kasturi SP, Kovalenkov Y, Rasheed AU, Yeiser P, Jinnah ZS, et al. Vaccine-induced plasmablast responses in rhesus macaques: phenotypic characterization and a source for generating antigen-specific monoclonal antibodies. J Immunol Methods (2015) 416:69-83. doi:10.1016/j.jim.2014.11.003

23. Demberg T, Brocca-Cofano E, Xiao P, Venzon D, Vargas-Inchaustegui D, Lee EM, et al. Dynamics of memory B-cell populations in blood, lymph nodes, and bone marrow during antiretroviral therapy and envelope boosting in simian immunodeficiency virus SIVmac251-infected rhesus macaques. J Virol (2012) 86:12591-604. doi:10.1128/JVI.00298-12

24. Neumann B, Klippert A, Raue K, Sopper S, Stahl-Hennig C. Characterization of $\mathrm{B}$ and plasma cells in blood, bone marrow, and secondary lymphoid organs of rhesus macaques by multicolor flow cytometry. J Leukoc Biol (2015) 97:19-30. doi:10.1189/jlb.1HI0514-243R

25. Martinez P, Sundling C, O’dell S, Mascola JR, Wyatt RT, Karlsson Hedestam GB. Primate immune responses to HIV-1 Env formulated in the saponin-based adjuvant AbISCO-100 in the presence or absence of TLR9 co-stimulation. Sci Rep (2015) 5:8925. doi:10.1038/srep08925

26. Spangberg M, Martinez P, Fredlund H, Karlsson Hedestam GB, Sundling C. A simple and safe technique for longitudinal bone marrow aspiration in cynomolgus and rhesus macaques. J Immunol Methods (2014) 408:137-41. doi:10.1016/j.jim.2014.05.004

27. Yammani RD, Haas KM. Primate B-1 cells generate antigen-specific B cell responses to T cell-independent type 2 antigens. J Immunol (2013) 190:3100-8. doi:10.4049/jimmunol.1203058

28. Hong S, Lee HW, Chang DY, You S, Kim J, Park JY, et al. Antibody-secreting cells with a phenotype of Ki-67low, CD138high, CD31high, and CD38high secrete nonspecific IgM during primary hepatitis A virus infection. J Immunol (2013) 191:127-34. doi:10.4049/jimmunol.1203540

29. Ellyard JI, Avery DT, Phan TG, Hare NJ, Hodgkin PD, Tangye SG. Antigenselected, immunoglobulin-secreting cells persist in human spleen and bone marrow. Blood (2004) 103:3805-12. doi:10.1182/blood-2003-09-3109

30. Shi W, Liao Y, Willis SN, Taubenheim N, Inouye M, Tarlinton DM, et al. Transcriptional profiling of mouse B cell terminal differentiation defines a signature for antibody-secreting plasma cells. Nat Immunol (2015) 16:663-73. doi:10.1038/ni.3154

31. Medina F, Segundo C, Campos-Caro A, Salcedo I, García-Poley A, Brieva JA. Isolation, maturational level, and functional capacity of human colon lamina propria plasma cells. Gut (2003) 52:383-9. doi:10.1136/gut.52.3.383
32. Govender D, Harilal P, Dada M, Chetty R. CD31 (JC70) expression in plasma cells: an immunohistochemical analysis of reactive and neoplastic plasma cells. J Clin Pathol (1997) 50:490-3. doi:10.1136/jcp.50.6.490

33. Fernandez JE, Deaglio S, Donati D, Beusan IS, Corno F, Aranega A, et al. Analysis of the distribution of human CD38 and of its ligand CD31 in normal tissues. J Biol Regul Homeost Agents (1998) 12:81-91.

34. Medina F, Segundo C, Brieva JA. Purification of human tonsil plasma cells: pre-enrichment step by immunomagnetic selection of $\mathrm{CD} 31+$ cells. Cytometry (2000) 99:231-4. doi:10.1002/(SICI)1097-0320(20000301)39:3< 231::AID-CYTO9>3.0.CO;2-G

35. Deaglio S, Morra M, Mallone R, Ausiello CM, Prager E, Garbarino G, et al. Human CD38 (ADP-ribosyl cyclase) is a counter-receptor of CD31, an Ig superfamily member. J Immunol (1998) 160:395-402.

36. Majid A, Lin T, Best G, Fishlock K, Hewamana S, Pratt G, et al. CD49d is an independent prognostic marker that is associated with CXCR4 expression in CLL. Leuk Res (2011) 35:750756. doi:10.1016/j.leukres.2010.10.022

37. Bo M, Tissino E, Benedetti D, Caldana C, Bomben R, Poeta G, et al. Microenvironmental interactions in chronic lymphocytic leukemia: the master role of CD49d. Semin Hematol (2014) 51:168176. doi:10.1053/j. seminhematol.2014.05.002

38. Cantor J, Browne CD, Ruppert R, Féral CC, Fässler R, Rickert RC, et al. CD98hc facilitates B cell proliferation and adaptive humoral immunity. Nat Immunol (2009) 10:412-9. doi:10.1038/ni.1712

39. Cantor JM, Ginsberg MH. CD98 at the crossroads of adaptive immunity and cancer. J Cell Sci (2012) 125:1373-82. doi:10.1242/jcs.096040

40. Mei HE, Yoshida T, Sime W, Hiepe F, Thiele K, Manz RA, et al. Blood-borne human plasma cells in steady state are derived from mucosal immune responses. Blood (2009) 113:2461-9. doi:10.1182/blood-2008-04-153544

41. Brandtzaeg P, Johansen FE. Mucosal B cells: phenotypic characteristics, transcriptional regulation, and homing properties. Immunol Rev (2005) 206:32-63. doi:10.1111/j.0105-2896.2005.00283.x

42. Fritz JH, Rojas O, Simard N, McCarthy DD, Hapfelmeier S, Rubino S, et al. Acquisition of a multifunctional IgA+ plasma cell phenotype in the gut. Nature (2011) 481:199-203. doi:10.1038/nature10698

43. Gommerman JL, Rojas OL, Fritz JH. Re-thinking the functions of IgA(+) plasma cells. Gut Microbes (2014) 5:652-62. doi:10.4161/19490976.2014.96 9977

44. Purtha WE, Tedder TF, Johnson S, Bhattacharya D, Diamond MS. Memory B cells, but not long-lived plasma cells, possess antigen specificities for viral escape mutants. J Exp Med (2011) 208:2599-606. doi:10.1084/jem.20110740

45. Tarlinton D, Good-Jacobson K. Diversity among memory B cells: origin, consequences, and utility. Science (2013) 341:1205-11. doi:10.1126/science. 1241146

46. Lavinder JJ, Wine Y, Giesecke C, Ippolito GC, Horton AP, Lungu OI, et al. Identification and characterization of the constituent human serum antibodies elicited by vaccination. Proc Natl Acad Sci U S A (2014) 111:2259-64. doi:10.1073/pnas.1317793111

47. Montezuma-Rusca JM, Moir S, Kardava L, Buckner CM, Louie A, Kim LJ, et al. Bone marrow plasma cells are a primary source of serum HIV-1-specific antibodies in chronically infected individuals. J Immunol (2015) 194:2561-8. doi:10.4049/jimmunol.1402424

48. Kyu SY, Kobie J, Yang H, Zand MS, Topham DJ, Quataert SA, et al. Frequencies of human influenza-specific antibody secreting cells or plasmablasts post vaccination from fresh and frozen peripheral blood mononuclear cells. J Immunol Methods (2009) 340:42-7. doi:10.1016/j.jim.2008.09.025

Conflict of Interest Statement: The authors declare that the research was conducted in the absence of any commercial or financial relationships that could be construed as a potential conflict of interest.

Copyright ( 2016 Martinez-Murillo, Pramanik, Sundling, Hultenby, Wretenberg, Spångberg and Karlsson Hedestam. This is an open-access article distributed under the terms of the Creative Commons Attribution License (CC BY). The use, distribution or reproduction in other forums is permitted, provided the original author(s) or licensor are credited and that the original publication in this journal is cited, in accordance with accepted academic practice. No use, distribution or reproduction is permitted which does not comply with these terms. 